\title{
Mathematical models as practical tool for road fatalities forecasts on road network in regions
}

\author{
Joanna Wachnicka ${ }^{1, *}$ \\ ${ }^{1}$ Gdansk University of Technology, Highway and Transportation Engineering Department, 80-233 \\ Gdansk, 11 Narutowicza Str., Poland
}

\begin{abstract}
The analysis of national data on the number of deaths showed that in Poland from 2010 to 2016 it was possible to reduce the number of fatalities by about $22 \%$. The tendency of changes in the number of fatalities, however, is not homogeneous. When data of individual voivodships is analyzed, the situation is different. The largest reduction in fatalities in the analyzed period of time concerned voivodship Świętokrzyskie, where there was more than $45 \%$ drop in the number of fatalities. The following voivodships: Łódzkie, Lubelskie and Podkarpackie recorded a decrease in over $30 \%$ of fatalities. Unfortunately, at the end of the classification there were four voivodships with a fall below $8 \%$, and what is disturbing voivodship Lubuskie recorded a nearly $5 \%$ increase in the number of fatalities. The current traffic safety management at the level of voivodships is often the implementation of central recommendations, which, as results from the analysis of statistical data, are not equally effective in every province. Therefore, models for forecasting changes in road safety are required. Taking into account local characteristics and implemented actions can be used to manage security more effectively at the regional level. This paper presents examples of the use of mathematical models to predict the number of fatalities in individual voivodships depending on the adopted action scenarios. Regression models were developed, taking into account demographic, infrastructural, economic and automotive factors. It turned out that in individual voivodships, various factors affect the level of road safety on roads differently. Therefore, an individual approach to each voivodship is important in order to reliably forecast the level of security.
\end{abstract}

\section{Introduction}

According to the latest data provided by the WHO (World Health Organization) of January 2018, more than 1.25 million people are still killed each year in road accidents. Death as a result of injuries in a traffic accident is the leading cause of death among people aged 15-29. At the same time, $90 \%$ of fatalities are people from countries with low levels of economic development or developing countries, although at the same time there are only $54 \%$ of all vehicles in the world. Almost half of the victims are unprotected road users. Road accidents cost most of the countries 3\% of their gross domestic product. Without continuous and

${ }^{*}$ Corresponding author: joanna.wachnicka@pg.edu.pl 
effective action until 2030, road accidents will continue to be the leading seven causes of death in the world. The new Sustainable Development Agenda adopted by 2030 assumes a $50 \%$ reduction in the number of deaths and injuries by 2020 [1]. The analysis of national data on the number of fatalities has shown that in Poland from 2010 to 2016 it was possible to reduce the number of fatalities by about $22 \%$. The tendency of changes in the number of fatalities, however, is not homogeneous. When data is analyzed in individual voivodships, the situation is different. The largest reduction in fatalities in the analyzed period of time concerned the province Świętokrzyski, where there was a more than $45 \%$ drop in the number of fatalities. The following provinces: Łódzkie, Lubelskie and Podkarpackie recorded a decrease in over $30 \%$ of fatalities. Unfortunately, at the end of the classification there were four voivodships with a fall below $8 \%$, and what is disturbing one Lubuskie voivodship recorded a nearly $5 \%$ increase in the number of fatalities. The current management of traffic safety at the level of voivodships is often the implementation of central recommendations resulting from the road safety improvement programs, However, according to the analysis of statistical data, it does not give uniform results in each of the provinces.

\section{Objectives}

The purpose of this article was to develop mathematical descriptive models characterizing the level of road safety in selected voivodships. The components of the model were to be characteristics describing the specific economic, automotive, infrastructural, geographical or social characteristics of a given voivodship, as well as taking into account systemic, preventive or supervisory activities in a given region. Such mathematical models could be a good tool for more effective road safety management in individual provinces. These models could be used as a tool for predicting, for example, the number of fatalities in individual voivodships depending on the adopted action scenarios. Thanks to this, it is possible to analyze what are the possibilities of reducing fatalities depending on the dynamics of changes in factors affecting this indicator. The assumed dynamics of changes in independent variables results from the current trend of changes in individual regions.

\section{Method}

Due to the diversity of voivodships in terms of size and population, it was decided that the fatalities $F_{i}$ models will be based on the social risk model, where the number of fatalities is calculated as the product of the probability of being a victim of a car accident in a region (province) $R F R_{i}^{w}$ and exposure to this risk, expressed as the population size of the $P_{i}$ region concerned. Due to the fact that voivodships in their present shape exist only from 1999 and, therefore, the number of modeling data is limited, it was decided to create baseline models $R F R_{i}^{k}$ constructed on data from all voivodships in Poland, and then calibrate such created models by multiplying the created base function $R F R_{i}^{k}$ times the modifying function created on the basis of data from a single, selected region $C R F R_{i}^{w}$, containing factors more strongly associated with the modeled variable in a given province than on average throughout the country.

$$
\begin{gathered}
F_{i}^{w}=R F R_{i}^{w} \cdot P_{i} \\
R F R_{i}^{w}=R F R_{i}^{k} \cdot C R F R_{i}^{W}
\end{gathered}
$$

where:

$F_{i}^{w}$ - number of fatalities [number] 
$P_{i}$ - population of region [100 thous. people],

$R F R_{i}^{W}$ - model of road fatalities rate in analyzed region [fatalities/100 thous. people],

$R F R_{i}^{k}$ - model of road fatalities rate in analyzed group of regions [fatalities/100 thous. people],

$C R F R_{i}^{W}$ - regional modification model of road fatalities rate.

This approach helps to build reliable models and, using the region's forecasted population, calculate the forecasted number of fatalities in relation to specific factors.

The influence of individual independent variables on the relative fatalitities rate index was analyzed using various methods of mathematical analysis, such as cross-correlation analysis of linear correlation, advanced analysis of the influence of linear and non-linear independent variables on the modeled variable using unique Data Mining algorithms, as well as functional analyzes the influence of independent variables on the variable RFR.

\section{Results}

\subsection{Mathematical model for all provinces in Poland}

As a result of the analyzes, predefined independent variables have been selected that have a strong link to the modeled relative indicator of the number of fatalities RFR:

- expected life lenght rate LEI [years],

- population density DP [people $/ \mathrm{km}^{2} /$ year],

- $\quad$ share of population leaving in the city PUP [\%],

- $\quad$ share of build up areas PUA [\%],

- individual gross domestic product GDPPC [thous.ID/person/year],

- population density of all roads in total $\mathrm{DR}_{(\mathrm{D})}$ [thous. $\mathrm{km} / 100$ thous.people/year],

- density of roads in total $\mathrm{DR}_{(\mathrm{A})}[\mathrm{km} / \mathrm{km} 2 /$ year $]$,

- demographic density of highways and expressways $\mathrm{DME}_{(\mathrm{D})}$ [thous. $\mathrm{km} / 100$ thous. people/year],

- expenditure on provincial/voivodship roads REXV [mln zł/ $/ \mathrm{km} /$ year],

- number of hospital emergency departments HEW [number/year],

- motorisation rate MRV [veh./person/year].

The obtained results are in line with the results of research in the literature, which was foreseen during the work on the issue. It also pointed to the impact of even such factors as the motorisation rate [2,3], the share of urbanized areas [4] or the share of highways [5] in the entire road network.

During the work on the models, it turned out that the Mazowieckie Voivodeship is very different from other provinces. While with the growth of the unitary national product in other voivodships, the level of road traffic safety is decreasing, in the Mazowieckie voivodship this decrease is not so unambiguous. In addition, the level of road safety in this region is significantly higher than the other provinces. The Mazowieckie Voivodeship is a specific voivodship in comparison to other regions of Poland, as shown in the preliminary analyzes carried out in the doctoral thesis [6]. It is a province with a clearly larger gross domestic product and the number of vehicles. It can be presumed that a very large percentage of people live there in cities, but at the same time perform a lot of travel during the day. Due to housing prices and spatial development, there are offices and other workplaces in the center of Warsaw, and "bedrooms" have been created on the outskirts of the city. This is a typical layout for large metropolises. A lot of travel, however, is done by individual transport. 
The registered population does not reflect the real picture of reality. Many people rent apartments in the capital, being officially a resident of another province. The above characteristics are not possible to be included in the quantitative variable due to the lack of reliable data. Therefore, it was decided to heuristically introduce the qualitative variable $\mathrm{S}$, which for the Mazowieckie Voivodship is 10, and for the other voivodships the value of 1 . Such a procedure proved to be a very good idea, because it was possible to include all data from the base to build the model and create a universal model for all voivodships. Below is a mathematical model developed along with graphical interpretation of the influence of one of the selected $\mathrm{DME}(\mathrm{D})$ variables.

$$
\begin{aligned}
& R F R_{i}^{k}=14447.86 \cdot S^{0,23} \cdot G D P P C^{-0,422} \cdot \exp (-0.08 \cdot L E I+ \\
& 0.37 \cdot D R_{(D)}-0.352 \cdot P U P-0.907 \cdot R E X V+0.02 \cdot P U A- \\
& \left.27.27 \cdot D M E_{(D)}\right), \mathrm{R}^{2}=0.7
\end{aligned}
$$

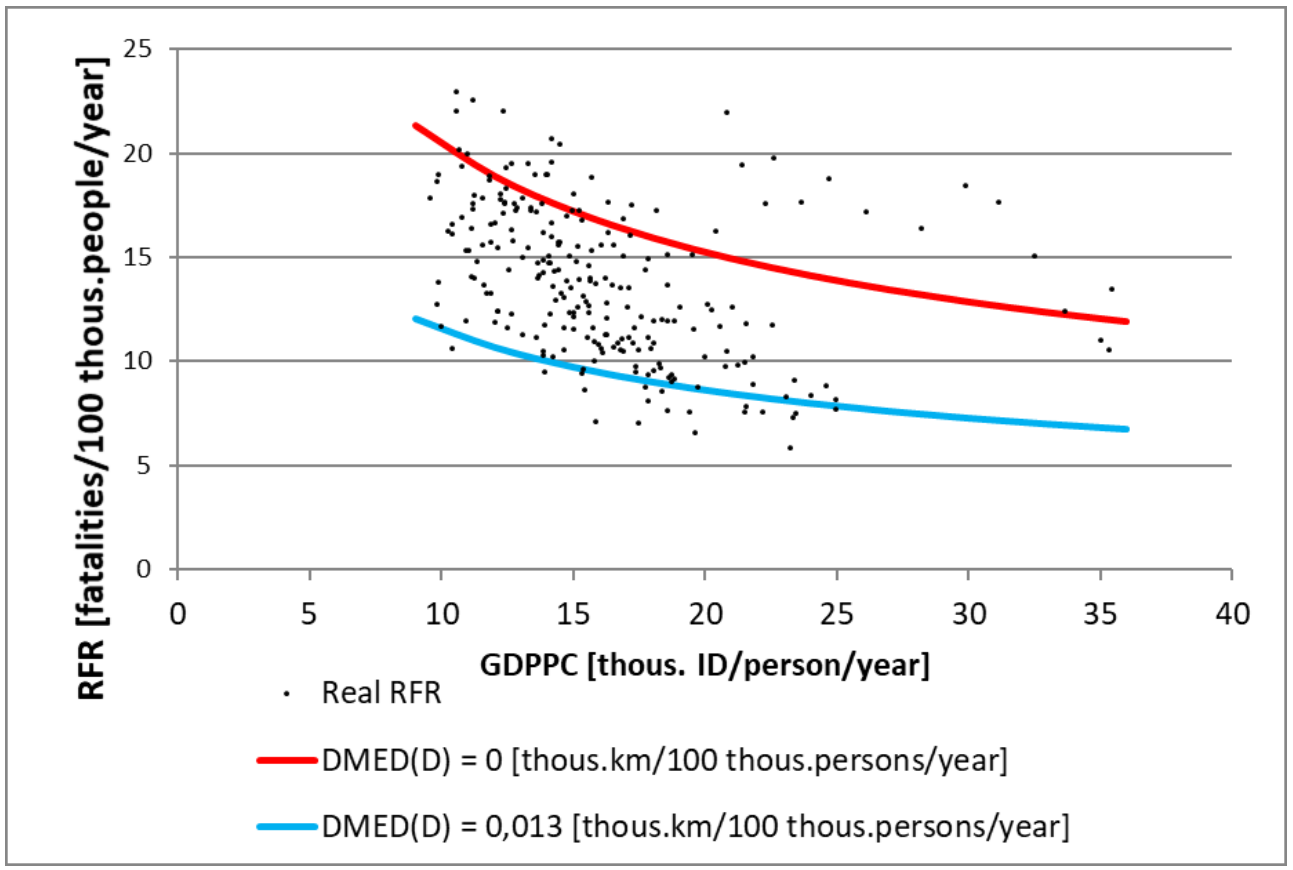

Fig. 1. The dependence of the modeled indicator of the number of fatalities in RFR on the unitary GDPPC and demographic density of highways and expressways $\mathrm{DME}_{(\mathrm{D})}$ against the background of real data. Model for all voivodships.

According to the adopted methodology, models of the relative fatalities rate in selected voivodships were developed. It turned out that depending on the province, individual factors had a different impact on the modeled variable RFR and it was possible to create models better suited by using a calibration function containing more data. Next, using the data on the forecast population in a given voivodeship, made available by the Central Statistical Office, it was possible to develop forecasts of the number of fatalities in a selected region depending on changes in individual components of the model. Below are examples of models for two selected voivodships, together with forecasts depending on the adopted scenario. The value of $\mathrm{S}$ for these provinces is 1 . 


\subsection{Forecasts for the Pomeranian Voivodship}

The first province selected to calibrate the model created for data from all provinces was the Pomeranian Voivodeship. In the case of this province, it is bigger importantance of demographic density of highways and expressways $\mathrm{DME}_{(\mathrm{D})}$ than in basic model $R F R_{i}^{k}$. The measure of goodness of the calibrated model for the Pomeranian Voivodship amounted to $95 \%$.

$$
\begin{aligned}
& F_{i}^{w}=P \cdot 14447.85 \cdot S^{0,23} \cdot G D P P C^{-0,42} \cdot \exp (-0.07 \cdot L E I+0.37 \cdot D R(D)- \\
& 0.91 \cdot R E X V+0.02 \cdot P U A-0.35 \cdot P U P-211.31 \cdot D M E(D)), \mathrm{R}^{2}=0.95
\end{aligned}
$$

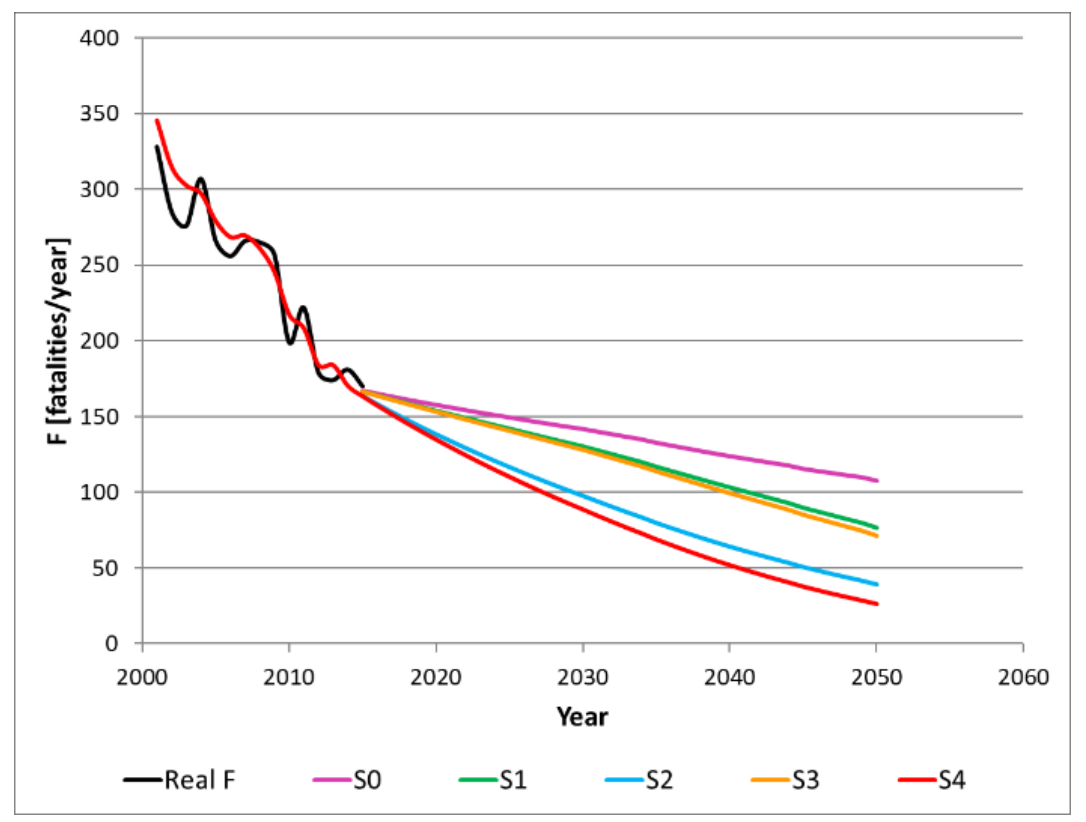

Fig. 2. The predicted number of fatalities $F$ depending on the assumed scenarios of changes in independent variables. Pomeranian Voivodeship.

Table 1 Assumed variability of selected factors in particular scenarios presented in Figure 2.

\begin{tabular}{|l|l|l|l|l|l|l|l|}
\hline \multirow{2}{*}{ Scenario } & \multicolumn{7}{|c|}{ Variable } \\
\cline { 2 - 7 } & GDPPC & LEI & DR(D) & REXV & PUA & PUP & DME(D) \\
\hline S0 & $+3 \%$ & const. & const. & const. & const. & const. & const. \\
\hline S1 & $+3 \%$ & const. & const. & $+10 \%$ & const. & const. & const. \\
\hline S2 & $+3 \%$ & $+0.3 \%$ & const. & const. & const. & const. & const. \\
\hline S3 & $+3 \%$ & const. & const. & const. & const. & const. & $+5 \%$ \\
\hline S4 & $+3 \%$ & $+0.3 \%$ & const. & $+10 \%$ & const. & const. & $+5 \%$ \\
\hline
\end{tabular}

As you can see, in the case of the province Pomerania steady economic growth, increased investments on voivodeship roads and increasing the number of motorways and expressways is the chance of a very large reduction in the number of fatalities in the province. Pomeranian until 2050 (Scenario S4). In this scenario a further increase in GDPPC at 3\% per annum was assumed, as well as an increase in life expectancy of $0.3 \%$ per year. In this scenario, a further increase of subsidies for provincial roads by $10 \%$ in relation to the previous year and also a $5 \%$ increase in demographic density of highways and expressways DME (D) per year was assumed. 


\subsection{Forecasts for the Kuyavian-Pomeranian Voivodeship}

The second province selected to calibrate the model created for data from all provinces was the Kuyavian-Pomeranian Voivodeship. In the case of this voivodship, statistically significant importance of such additional variables as expenditure on provincial/voivodship roads REXV, percentage of highways and express roads PME and density of vehicles in total DV. This may be due to the rapid and dynamic development of the highest-class road network in this province in recent times. The measure of goodness of the calibrated model for the Pomeranian Voivodship amounted to $79 \%$.

$$
\begin{aligned}
& F_{i}^{w}=P \cdot 14447.85 \cdot S^{0,23} \cdot G D P P C^{-0,42} \cdot \exp (-0.07 \cdot L E I+0.37 \cdot D R(D)-7.32 \cdot \\
& R E X V+0.02 \cdot P U A-0.35 \cdot P U P-27.27 \cdot D M E(D)-2.06 \cdot P M E+36.30 \cdot D V) \\
& \mathrm{R}^{2}=0.79
\end{aligned}
$$

Table 2 Assumed variability of selected factors in particular scenarios presented in Figure 3.

\begin{tabular}{|l|l|l|l|l|l|l|l|l|l|}
\hline \multirow{2}{*}{ Scenario } & \multicolumn{9}{|c|}{ Variable } \\
\cline { 2 - 10 } & GDPPC & LEI & DR(D) & REXV & PUA & PUP & DME(D) & PME & DV \\
\hline S0 & $+2 \%$ & const. & const. & const. & const. & const. & const. & const. & const. \\
\hline S1 & $+2 \%$ & const. & const. & $+5 \%$ & const. & const. & const. & const. & const. \\
\hline S2 & $+2 \%$ & $+0.3 \%$ & const. & stała & const. & const. & const. & const. & const. \\
\hline S3 & $+2 \%$ & const. & const. & stała & const. & const. & $+5 \%$ & const. & const. \\
\hline S4 & $+2 \%$ & $+0.3 \%$ & const. & stałe & const. & const. & const. & const. & $+0.6 \%$ \\
\hline S5 & $+2 \%$ & const. & const. & stała & const. & const. & const. & const. & $+0.6 \%$ \\
\hline S6 & $+2 \%$ & $+0.3 \%$ & const. & $+5 \%$ & const. & const. & $+5 \%$ & const. & const. \\
\hline
\end{tabular}

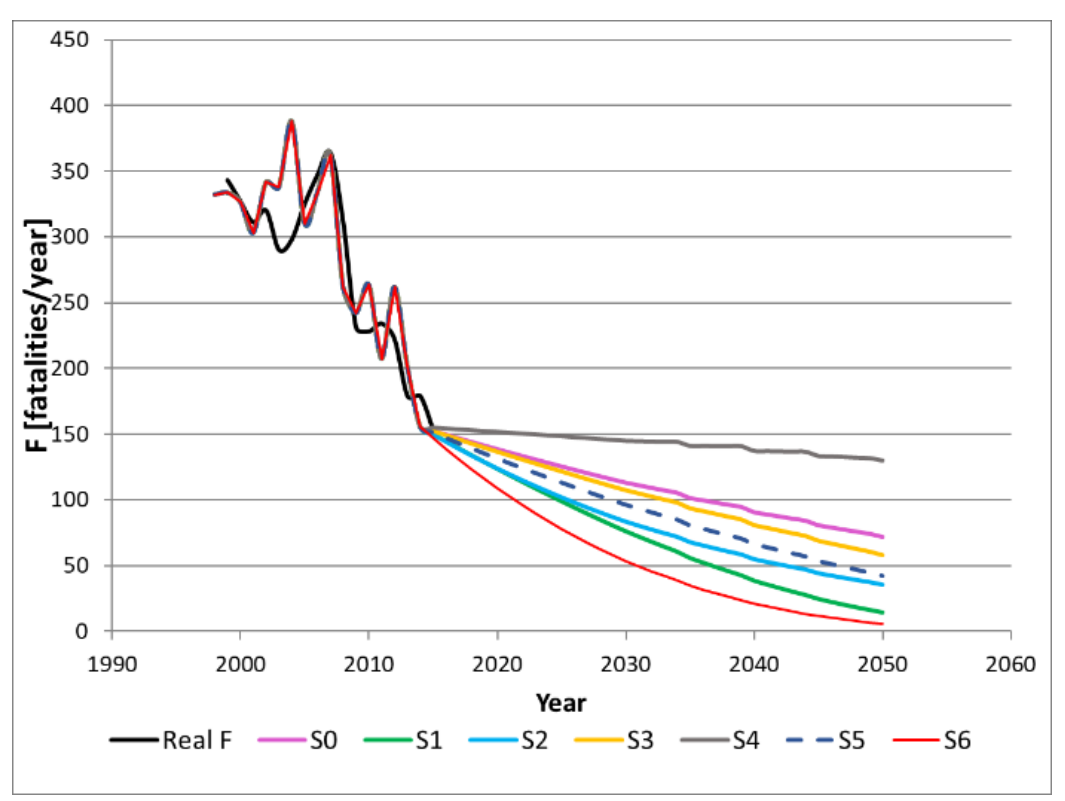

Fig. 3. The predicted number of fatalities $F$ depending on the assumed scenarios of changes in independent variables. Kuyavian-Pomeranian Voivodeship.

It turns out that in the province In Kujawsko-Pomorskie, there is even more potential for reducing the number of fatalities in road accidents. There is a chance to achieve almost zero vision in this region in 2050 (Scenario S6). In this scenario, it was assumed that in the Kujawsko-Pomorskie voivodship GDDPC will increase with the existing growth rate of $2 \%$ per annum, the life expectancy will increase by $0.3 \%$ per annum compared to the previous 
year, and the provincial road financing will grow by $5 \%$ annually and demographic density of highways and expressways $\mathrm{DME}(\mathrm{D})$ as well.

\section{Conclusion}

It is possible to create mathematical models describing changes of road safety in individual voivodships. It is important to collect data characterizing the region regularly. It is also important to have access to information on system activities as well as the operation of road safety related services: police, emergency services and traffic control. Perhaps these data are collected, however, they are not easily and widely available in any voivodship, which makes it impossible to develop models that take into account these characteristics. In addition, there is still a lack of data on road traffic on all roads of a given region. This is a key variable that would increase models' measure of the goodness and allow for even better forecasting.

\section{References}

1. WHO Fact Sheet 2018. Road traffic injuries. Available at: http://www.who.int/mediacentre/factsheets/fs358/en/, (2018)

2. S. Oppe, The development of traffic and traffic safety in six developed countries, Accid. Anal. Prev. 23 pp. 401-412 (1991). doi:10.1016/0001-4575(91)90059-E

3. J. Broughton, Predictive models of road accident fatalities, Traffic Eng. Control. 29 pp. 296-300 (1988)

4. S. Erdogan, Explorative spatial analysis of traffic accident statistics and road mortality among the provinces of Turkey., J. Safety Res. 40 pp. 341-351 (2009). doi:10.1016/j.jsr.2009.07.006

5. K. Jamroz, Highway Enginnering Risk Assessment, Arch. Transp. 19 pp. 67-74 (2007)

6. J. Wachnicka, Modelling risk measures on the road network in the regions. Phd Thesis (Politechnika Gdańska, Gdańsk, 2017) 